\title{
AN APPROXIMATION THEORY FOR GENERALIZED FREDHOLM QUADRATIC FORMS AND INTEGRAL-DIFFERENTIAL EQUATIONS
}

BY

\author{
J. GREGORY AND G. C. LOPEZ
}

\begin{abstract}
An approximation theory is given for a very general class of elliptic quadratic forms which includes the study of $2 n$th order (usually in integrated form), selfadjoint, integral-differential equations. These ideas follows in a broad sense from the quadratic form theory of Hestenes, applied to integraldifferential equations by Lopez, and extended with applications for approximation problems by Gregory.
\end{abstract}

The application of this theory to a variety of approximation problem areas in this setting is given. These include focal point and focal interval problems in the calculus of variations/optimal control theory, oscillation problems for differential equations, eigenvalue problems for compact operators, numerical approximation problems, and finally the intersection of these problem areas.

In the final part of our paper our ideas are specifically applied to the construction and counting of negative vectors in two important areas of current applied mathematics: In the first case we derive comparison theorems for generalized oscillation problems of differential equations. The reader may also observe the essential ideas for oscillation of many nonsymmetric (indeed odd order) ordinary differential equation problems which will not be pursued here. In the second case our methods are applied to obtain the "Euler-Lagrange equations" for symmetric tridiagonal matrices. In this significant new result (which will allow us to reexamine both the theory and applications of symmetric banded matrices) we can construct in a meaningful way, negative vectors, oscillation vectors, eigenvectors, and extremal solutions of classical problems as well as faster more efficient algorithms for the numerical solution of differential equations.

In conclusion it appears that many physical problems which involve symmetric differential equations are more meaningful presented as integral differential equations (effects of friction on physical processes, etc.). It is hoped that this paper will provide the general theory and present examples and methods to study integral differential equations.

Received by the editors August 27, 1973 and, in revised form, April 8, 1975. AMS (MOS) subject classifications (1970). Primary 45J05, 49 A30, 34C10.

Key words and phrases. Approximation theory, conjugate points, oscillations, calculus of variations, Fredholm integral differential equations, spline approximations. 
1. Introduction. The main purpose of this paper is to present an approximation theory of quadratic forms which is applicable to a very general class of quadratic forms and to linear selfadjoint operators of a generalized Fredholm type. That is $2 n$th order, integral-differential systems such as $\tau_{\beta}^{n}(t)=v_{\beta}^{n-1}(t)$, or as the generalized system:

$$
\frac{d^{n}}{d t^{n}}\left[\tau_{\beta}^{n}(t)\right]-\frac{d^{n-1}}{d t^{n-1}}\left[\tau_{\beta}^{n-1}(t)\right]+\ldots+(-1)^{n}\left[\tau_{\beta}^{0}(t)\right]=0,
$$

where $\operatorname{arcs} x(t)=\left(x_{1}(t), x_{2}(t), \ldots, x_{p}(t)\right)$ define equations

$$
\begin{aligned}
\tau_{\beta}^{j}(t) & =R_{\alpha \beta}^{i j}(t) x_{\alpha}^{(i)}(t)+\int_{a}^{b} K_{\alpha \beta}^{i j}(s, t) x_{\alpha}^{(i)}(s) d s, \\
v_{\beta}^{0}(t) & =\int_{a}^{t} \tau_{\beta}^{0}(s) d s+c_{\beta}^{0}, \\
v_{\beta}^{k}(t) & =\int_{a}^{t}\left[\tau_{\beta}^{k}(s)-v_{\beta}^{k-1}(s)\right] d s+c_{\beta}^{k}
\end{aligned}
$$

$(\alpha, \beta=1, \ldots, p ; k=1, \ldots, n-1 ; i, j=0, \ldots, n)$. In the above $R_{\alpha \beta}^{i j}(t)$, $K_{\alpha \beta}^{i j}(t)$ satisfy smoothness and symmetry properties sufficient to guarantee that our system is the Euler-Lagrange equation for an appropriate quadratic form; $x_{\alpha}^{(i)}(t)$ denotes the $i$ th derivative of the $\alpha$ th component function; and repeated indices are summed. For $p=1$ and $n=1$ we obtain (ignoring subscripts) the generalized equation

$$
\begin{array}{r}
\frac{d}{d t}\left[R^{i 1}(t) x^{(i)}(t)+\int_{a}^{b} K^{i 1}(s, t) x^{(i)}(s) d s\right] \\
=R^{i 0}(t) x^{(i)}(t)+\int_{a}^{b} K^{i 0}(s, t) x^{(i)}(s) d s
\end{array}
$$

where $i=0,1$. For $p=1$ we obtain the $2 n$th integral-differential equation (in generalized form)

$$
\frac{d^{n}}{d t^{n}}\left[\tau^{n}(t)\right]-\frac{d^{n-1}}{d t^{n-1}}\left[\tau^{n-1}(t)\right]+\frac{d^{n-2}}{d t^{n-2}}\left[\tau^{n-2}(t)\right]-\ldots+(-1)^{n} \tau^{0}(t)=0
$$

Applications of our theory to approximating problems dealing with eigenvalue problems, oscillation problems or focal point problems, and numerical problems will be considered.

The fundamental quadratic form theory was given by Hestenes in 1951 to handle recurring "second variation" problems in the calculus of variations. This theory was generalized by Gregory to an approximation theory of quadratic forms. In one sense this paper is an application of these ideas to a very general problem in differential equations.

The outline of this paper is as follows: In $\$ 2$ we present the theory of quadratic forms by Lopez. The connection between the quadratic form theory and the Euler-Lagrange equations, plus the transversality conditions discussed 
below, are the main results. In $\S 3$ we present the approximation theory of quadratic forms by Gregory which is sufficiently general to handle the quadratic forms in $\$ 2$. The main results are given in terms of inequalities involving nonnegative indices. In particular we show that the hypotheses for these inequalities are sufficiently general to include the "resolution spaces" of Hestenes [6] for focal point theories and "continuous" perturbations of coefficients of quadratic forms and integral-differential equations.

In $\S 4$ we extend the approximation setting of $\$ 3$ to obtain an approximate theory of focal points and focal intervals. These results are then interpreted to obtain existence theorems and other properties for $2 n$th order integral-differential equations systems. In $\$ 5$ we discuss in (a general way) how this theory may be applied to a multitude of problems. In particular we discuss in some detail the application to numerical focal point problems.

The inequalities such as (15) are used on three levels in this paper. The first level leads to a theory of quadratic forms with applications given by Hestenes [6] and Lopez [8]. The second level leads to an approximation theory for "level one" problems exemplified by Theorems 11 to 15 . A third level is a numerical approximation theory for the "level two" problems such as in $\S 5$.

The statements " $2 n$th order equations" or "generalized equations" refer to the integrated form $\tau_{\beta}^{n}(t)=v_{\beta}^{n-1}(t)$, or to the $n$th derivative of this expression if it exists.

2. Fredholm type quadratic forms. In this section we give the quadratic form theory leading to the integral-differential equations of $\$ 1$. A part of this section is found in [8]. This work is an "application" of the quadratic form theory of Hestenes [6].

The fundamental Hilbert space $A$ considered in this section is the set of functions $z(t)=\left[z_{1}(t), \ldots, z_{p}(t)\right]$ whose $\alpha$ th component, $z_{\alpha}(t)$, is a realvalued function defined on the interval $a \leqslant t \leqslant b$ of class $C^{n-1} ; z_{\alpha}^{(n-1)}(t)$ is absolutely continuous and $z_{\alpha}^{(n)}(t)$ is Lebesgue square integrable on $a \leqslant t \leqslant b$. The inner product is given by

$$
(x, y)=x_{\alpha}^{(k)}(a) y_{\alpha}^{(k)}(a)+\int_{a}^{b} x_{\alpha}^{(n)}(t) y_{\alpha}^{(n)}(t) d t
$$

where $\alpha=1, \ldots, p ; k=0, \ldots, n-1$; superscripts denote the order of differentiation; and repeated indices (except for $n$ ) are summed.

The fundamental quadratic form $J(x)$ is given by

$$
\begin{aligned}
J(x)= & H(x)+\int_{a}^{b} \int_{a}^{b} k_{\alpha \beta}^{i j}(s, t) x_{\alpha}^{(i)}(s) x_{\beta}^{(j)}(t) d s d t \\
& +\int_{a}^{b} R_{\alpha \beta}^{i j}(t) x_{\alpha}^{(i)}(t) x_{\beta}^{(j)}(t) d t
\end{aligned}
$$


$(\alpha, \beta=1, \ldots, p ; i, j=0, \ldots, n)$, where $R_{\alpha \beta}^{i j}(t)=R_{\beta \alpha}^{i i}(t)$ are essentially bounded and integrable functions on $a \leqslant t \leqslant b$;

$$
H(x)=A_{\alpha \beta}^{k l} x_{\alpha}^{(k)}(a) x_{\beta}^{(l)}(a),
$$

$A_{\alpha \beta}^{k l}=A_{\beta \alpha}^{l k}(k, l=0, \ldots, n-1)$ are $n^{2} p^{2}$ real numbers; $K_{\alpha \beta}^{i j}(s, t)=K_{\beta \alpha}^{i i}(t, s)$ are essentially bounded and integrable functions on $a \leqslant t \leqslant b$; and

$$
R_{\alpha \beta}^{n n}(t) \pi_{\alpha} \pi_{\beta} \geqslant h \pi_{\alpha} \pi_{\alpha}
$$

holds almost everywhere on $a \leqslant t \leqslant b$, for every $\pi=\left(\pi_{1}, \ldots, \pi_{p}\right)$ in $E^{p}$, and some $h>0$. This inequality is the ellipticity condition of Hestenes in this setting [6].

The connection between the quadratic forms and integral-differential equations is now given.

Let $B$ denote a subspace of $A$ such that $x$ is in $B$ if and only if

$$
L_{\gamma}(x)=M_{\gamma \alpha}^{k} x_{\alpha}^{(k)}(a)=0, \quad x_{\beta}^{(l)}(b)=0
$$

$(\alpha, \beta=1, \ldots, p ; k, l=0, \ldots, n-1 ; \gamma=1, \ldots, m \leqslant n p)$ where $M_{\gamma \alpha}^{k}$ are real numbers such that the linear functionals $L_{\gamma}(x)$ are linearly independent on A. Let $B(\lambda), a \leqslant \lambda \leqslant b$, denote the subspace of $B$ whose component functions satisfy

$$
x_{\alpha}^{(k)}(t) \equiv 0 \text { on } \lambda \leqslant t \leqslant b \text { for } k=0,1, \ldots, n-1 \text {. }
$$

For any $\operatorname{arc} x(t)$ in $A$ set

$$
\tau_{\beta}^{j}(t)=R_{\alpha \beta}^{i j}(t) x_{\alpha}^{(i)}(t)+\int_{a}^{b} K_{\alpha \beta}^{i j}(s, t) x_{\alpha}^{(i)}(s) d s
$$

for almost all $t$ on $a \leqslant t \leqslant b$. Define the recursive relations

(7a) $v_{\beta}^{0}(t)=\int_{a}^{t} \tau_{\beta}^{0}(s) d s+c_{\beta}^{0}$,

(7b) $v_{\beta}^{k}(t)=\int_{a}^{t}\left[\tau_{\beta}^{k}(s)-v_{\beta}^{k-1}(s)\right] d x+c_{\beta}^{k} \quad(k=1, \ldots, n-1)$,

where $c_{\beta}^{0}, \ldots, c_{\beta}^{n-1}$ are real numbers. Let $J(x, y)$ be the bilinear form associated with $J(x)$, i.e. $J(x)=J(x, x)$.

THEOREM 1. Let $J(x)$ be the quadratic form given by (2). There exists an arc $x=\left(x_{1}(t), \ldots, x_{p}(t)\right)$ in A such that $J(x, y)=0$ for all $y=\left(y_{1}(t)\right.$, $\left.\ldots, y_{p}(t)\right)$ in $B(\lambda)$ if and only if the constants $c_{\beta}^{0}, \ldots, c_{\beta}^{n-1}$ in (7) and constants $\mu_{1}, \ldots, \mu_{m}$ can be chosen such that the Euler equations

$$
\tau_{\beta}^{n}(t)=v_{\beta}^{n-1}(t) \quad(\beta=1, \ldots, p)
$$

hold almost everywhere on $a \leqslant t \leqslant \lambda$, and the transversality conditions 


$$
A_{\alpha \beta}^{k l} x_{\alpha}^{(k)}(a)+\mu_{\gamma} M_{\gamma \beta}^{l}-v_{\beta}^{l}(a)=0
$$

hold at $t=a$.

The proof of this result follows in the expected way from the method of integration by parts or by the Riesz Representation Theorem for Hilbert spaces. For $n=1$ and $K_{\alpha \beta}^{i j}(s, t)$ identically zero for all indices, the results are given in Hestenes [6, pp. 535, 541, 542].

We remark that $[10]$ gives a more general theorem and includes the complex case. We also note that (8) is the integrated form of the $2 n$th order integral-differential equation (if it exists)

$$
\frac{d^{n}}{d t^{n}}\left[\tau_{\beta}^{n}(t)\right]-\frac{d^{n-1}}{d t^{n-1}}\left[\tau_{\beta}^{n-1}(t)\right]+\cdots+(-1)^{n} \tau_{\beta}^{0}(t)=0 .
$$

3. The approximation theory. In this section we state the fundamental approximation hypothesis. As examples of this hypothesis we show (in Theorem 8) that our hypothesis is satisfied by approximation quadratic forms on the space $B$ of $\S 2$ defined by (5a). In Theorem 10 we show that our hypothesis is satisfied by the "resolution spaces" $B(\lambda)$ of $(5 b)$.

We now state the approximation hypothesis given in [1]. These hypotheses are contained in conditions (11) and (12). In this section A will denote a Hilbert space with inner product $(x, y)$ and norm $\|x\|=(x, x)^{1 / 2}$. Strong convergence will be denoted by $x_{q} \Rightarrow x_{0}$ and weak convergence by $x_{q} \rightarrow x_{0}$. The bilinear forms $Q(x, y)$ in this paper are assumed to be bounded and symmetric. The associated quadratic form is given by $Q(x)=Q(x, x)$.

Let $\Sigma$ be a metric space with metric $\rho$. A sequence $\left\{\sigma_{r}\right\}$ in $\Sigma$ converges to $\sigma_{0}$ in $\Sigma$, written $\sigma_{r} \rightarrow \sigma_{0}$, if $\lim _{r=\infty} \rho\left(\sigma_{r}, \sigma_{0}\right)=0$. For each $\sigma$ in $\Sigma$ let $\mathrm{A}(\sigma)$ be a closed subspace of $\mathrm{A}$ such that

(11a) if $\sigma_{r} \rightarrow \sigma_{0}, x_{r}$ in $A\left(\sigma_{r}\right), x_{r} \rightarrow y_{0}$ then $y_{0}$ is in $A\left(\sigma_{0}\right)$;

(11b) if $x_{0}$ is in $A\left(\sigma_{0}\right)$ and $\epsilon>0$ there exists $\delta>0$ such that whenever $\rho\left(\sigma, \sigma_{0}\right)<\delta$, there exists $x_{\sigma}$ in $A(\sigma)$ satisfying $\left\|x_{0}-x_{\sigma}\right\|<\epsilon$.

For each $\sigma$ in $\Sigma$ let $J(x ; \sigma)$ be a quadratic form defined on $A(\sigma)$ with $J(x, y ; \sigma)$ the associated bilinear form. For $r=0,1,2, \ldots$ let $x_{r}$ be in $A\left(\sigma_{r}\right)$, $y_{r}$ in $A\left(\sigma_{r}\right)$ such that: if $x_{r} \rightarrow x_{0}, y_{r} \Rightarrow y_{0}$ and $\sigma_{r} \rightarrow \sigma_{0}$ then

$$
\begin{aligned}
& \lim _{r=\infty} J\left(x_{r}, y_{r} ; \sigma_{r}\right)=J\left(x_{0}, y_{0} ; \sigma_{0}\right) ; \\
& \lim _{r=\infty} \inf J\left(x_{r} ; \sigma_{r}\right) \geqslant J\left(x_{0} ; \sigma_{0}\right) ; \\
& \lim _{r=\infty} J\left(x_{r} ; \sigma_{r}\right)=J\left(x_{0} ; \sigma_{0}\right) \text { implies } x_{r} \Rightarrow x_{0} .
\end{aligned}
$$

The form $J(x)$ is elliptic on $A$ if conditions (12b) and (12c) hold with 
$J(x)$ replacing $J(x ; \sigma)$ and $A$ replacing $A(\sigma)$. The signature (index) of $Q(x)$ on a subspace $D$ of $A$ is the dimension of a maximal, linear subclass $E$ of $D$ such that $x \neq 0$ in $E$ implies $Q(x)<0$. The nullity of $Q(x)$ on $D$ is the dimension of the space $D_{0^{*}}=\{x$ in $D \mid Q(x, y)=0$ for all $y$ in $D\}$. It can be shown that the sum of the index and nullity is the dimension of a maximal subclass $F$ of $D$ such that $x \neq 0$ in $F$ implies $Q(x) \leqslant 0$. In this paper we denote the index and nullity of $J(x ; \sigma)$ on $A(\sigma)$ by $s(\sigma)$ and $n(\sigma)$ respectively. Finally the bilinear form $Q(x, y)$ is compact if $x_{q} \rightarrow x_{0}$ and $y_{q} \rightarrow y_{0}$ implies $Q\left(x_{q}, y_{q}\right) \rightarrow Q\left(x_{0}, y_{0}\right)$. $Q(x)$ is compact if $Q(x, y)$ is.

Theorems 2 to 5 have been given in [1]

THEOREM 2. Assume conditions (11a), (12b) and (12c) hold. Then for any $\sigma_{0}$ in $\Sigma$ there exists $\delta>0$ such that $\rho\left(\sigma_{0}, \sigma\right)<\delta$ implies

$$
s(\sigma)+n(\sigma) \leqslant s\left(\sigma_{0}\right)+n\left(\sigma_{0}\right) .
$$

THEOREM 3. Assume conditions (11b) and (12a) hold. Then for any $\sigma_{0}$ in $\Sigma$ there exists $\delta>0$ such that $\rho\left(\sigma_{0}, \sigma\right)<\delta$ implies

$$
s(\sigma) \leqslant s(\sigma) \text {. }
$$

Combining Theorems 2 and 3 we obtain

THEOREM 4. Assume conditions (11) and (12) hold. Then for any $\sigma_{0}$ in $\Sigma$ there exists $\delta>0$ such that $\rho\left(\sigma, \sigma_{0}\right)<\delta$ implies

$$
s\left(\sigma_{0}\right) \leqslant s(\sigma) \leqslant s(\sigma)+n(\sigma) \leqslant s\left(\sigma_{0}\right)+n\left(\sigma_{0}\right) .
$$

Corollary 5. Assume $\delta>0$ has been chosen such that $\rho\left(\sigma, \sigma_{0}\right)<\delta$ implies inequality (15) holds. Then if $\rho\left(\sigma, \sigma_{0}\right)<\delta$ we have

$$
\begin{gathered}
n(\sigma) \leqslant n\left(\sigma_{0}\right), \\
n(\sigma)=n\left(\sigma_{0}\right) \text { implies } s(\sigma)=s\left(\sigma_{0}\right), \\
n\left(\sigma_{0}\right)=0 \text { implies } s(\sigma)=s\left(\sigma_{0}\right) \text { and } n(\sigma)=0 .
\end{gathered}
$$

In the remaining part of this section we show that problems of $\S 2$ satisfy the hypothesis given at the beginning of this section and hence Theorems 2 to 5. Theorem 6 characterizes weak and strong convergence in A with inner product given by (1). This result is the expected generalization given by Hestenes [6, Lemma 4.1] for $n=1$ and $K_{\alpha \beta}^{i j}(s, t)$ identically zero for all indices. Theorem 7 describes the compact (weakly continuous) quadratic forms associated with $J(x)$ given by (2) and contains one of the fundamental ideas of elliptic or Legendre forms given by Hestenes [6, Theorem 11.6]. Both theorems are given in [8]. In Theorem 6 let $\alpha=1, \ldots, p$ and $k=0, \ldots, n-1$; then we have 
THEOREM 6. The relation $x_{q}=\left[x_{q 1}(t), x_{q 2}(t), \ldots, x_{q p}(t)\right]$ converges strongly to $x_{0}=\left[x_{01}(t), x_{02}(t), \ldots, x_{0 p}(t)\right]$, denoted by $x_{q} \Rightarrow x_{0}$, holds if and only if, for each $\alpha$ and $k, x_{q \alpha}^{(k)}(0) \rightarrow x_{0 \alpha}^{(k)}(0)$ and $x_{q \alpha}^{(n)}(t) \rightarrow x_{0 \alpha}^{(n)}(t)$ in the mean of order two. Similarly $x_{q}$ converges weakly to $x_{0}$, denoted by $x_{q} \rightarrow x_{0}$, holds if and only if, for each $\alpha$ and $k, x_{q \alpha}^{(k)}(0) \rightarrow x_{0 \alpha}^{(k)}(0)$ and $x_{q \alpha}^{(n)}(t) \rightarrow x_{0 \alpha}^{(n)}(t)$ weakly in the class of Lebesgue square summable functions. In either case for each $\alpha$ and $k, x_{q \alpha}^{(k)}(t) \rightarrow x_{q 0}^{(k)}(t)$ uniformly on $a \leqslant t \leqslant b$.

THEOREM 7. If $J(x)$ is given in (2) and

$$
D(x)=\int_{a}^{b} R_{\alpha \beta}^{n n}(t) x_{\alpha}^{(n)}(t) x_{\beta}^{(n)}(t) d t
$$

then $K(x)=J(x)-D(x)$ is compact.

We note that $K(x)$ is $J(x)$ except for the terms which give rise to the principal part

$$
R_{\alpha \beta}^{n n}(t) \frac{d^{2 n}}{d t^{2 n}} x_{\alpha}(t) \quad(\alpha, \beta=1, \ldots, p)
$$

of equation (10). Furthermore $D(x)$ is elliptic (in the sense of Hestenes) and generates a norm on $B$ equivalent to (1). If $K_{1}(x)$ is any compact form then $K_{1}(x)+D(x)$ is elliptic and thus has finite index and nullity.

In the remainder of this section we assume for each $\sigma$ in a metric spaces $(\Sigma, \rho)$ quadratic forms of the type

$$
\begin{aligned}
J(x ; \sigma)= & A_{\alpha \beta \sigma}^{k l} x_{\alpha}^{(k)}(a) x_{\beta}^{(l)}(a) \\
& +\int_{a}^{b} \int_{a}^{b} K_{\alpha \beta \sigma}^{i j}(s, t) x_{\alpha}^{(i)}(s) x_{\beta}^{(j)}(t) d s d t \\
& +R_{\alpha \beta \sigma}^{i j}(t) x_{\alpha}^{(i)}(t) x_{\beta}^{(j)}(t) d t
\end{aligned}
$$

where the indices $\alpha, \beta, k, l, i$, and $j$, symmetry and smoothness properties of $A_{\alpha \beta \sigma}^{k l}, K_{\alpha \beta \sigma}^{i j}$, and $R_{\alpha \beta \sigma}^{i j}$ are those associated with equation (2). In order to illustrate later developments we will show that conditions (12) hold if the coefficients $A_{\alpha \beta \sigma}^{k l}, K_{\alpha \beta \sigma}^{i j}(s, t)$, and $R_{\alpha \beta \sigma}(t)$ of (18) are continuous in $\sigma$ and continuous (for fixed $\sigma$ ) in the independent variables $t$ and $s$. We will also show that conditions (11) hold on "resolution subspaces" $B(\lambda)$ of $B$.

THEOREM 8. Let $B$ denote the space of functions given by (5). Let $A_{\alpha \beta \sigma}^{k l}$, $K_{\alpha \beta \sigma}^{i j}(s, t)$, and $R_{\alpha \beta \sigma}(t)$ satisfy the continuity properties given above. Then conditions (12) hold on $B$.

For (12a), if $x_{r} \rightarrow x_{0}, y_{r} \Rightarrow y_{0}$ and $\sigma_{r} \rightarrow \sigma_{0}$ then

$$
\begin{aligned}
\left|J\left(x_{r}, y_{r} ; \sigma_{r}\right)-J\left(x_{0}, y_{0} ; \sigma_{0}\right)\right| \leqslant & \left|J\left(x_{r}, y_{r} ; \sigma_{r}\right)-J\left(x_{r}, y_{r} ; \sigma_{0}\right)\right| \\
& +\left|J\left(x_{r}, y_{r} ; \sigma_{0}\right)-J\left(x_{0}, y_{0} ; \sigma_{0}\right)\right| .
\end{aligned}
$$


The second term becomes arbitrarily small as $J\left(x, y ; \sigma_{0}\right)$ is elliptic on $B$. That is it can be expressed as the difference $D(x, y)-K(x, y)$ where $D$ is topologically equivalent to the inner product $(x, y)$, and $K(x, y)$ is compact. Equivalently the second term becomes small by use of Theorem 6 since the coefficients are fixed.

The first term is bounded by $M_{1} \psi(\sigma)\left\|x_{r}\right\|\left\|y_{r}\right\| \leqslant M_{2} \psi(\sigma)$ where

$\psi(\sigma)=3 \sup \left\{\left|R_{\alpha \beta \sigma_{0}}^{i j}(t)-R_{\alpha \beta \sigma}^{i j}(t)\right|,\left|K_{\alpha \beta \sigma_{0}}^{i j}(s, t)-K_{\alpha \beta \sigma}^{i j}(s, t)\right|,\left|A_{\alpha \beta \sigma_{0}}^{k l}-A_{\alpha \beta \sigma}^{k l}\right|\right\}$ and the supremum is taken for $s, t$ in $[a, b] ; \alpha, \beta=1, \ldots, p ; i, j=0, \ldots, n$; $k, l=0, \ldots, n-1$. Thus the first difference tends to zero as $\sigma \rightarrow \sigma_{0}$ by the continuity of the coefficients and the fact that both weak and strong convergence implies boundedness by Theorem 6 .

Similar arguments for (12b) and (12c) hold. For example, if $x_{r} \rightarrow x_{0}$ then

$$
J\left(x_{r} ; \sigma_{r}\right)-J\left(x_{0} ; \sigma_{0}\right)=J\left(x_{r} ; \sigma_{r}\right)-J\left(x_{r} ; \sigma_{0}\right)+J\left(x_{r} ; \sigma_{0}\right)-J\left(x_{0} ; \sigma_{0}\right) .
$$

Since

$$
\left|J\left(x_{r} ; \sigma_{r}\right)-J\left(x_{r} ; \sigma_{0}\right)\right| \leqslant M_{3} \psi\left(\sigma_{r}\right) \rightarrow 0 \text { as } \sigma_{r} \rightarrow \sigma_{0}
$$

(12b) holds since $J\left(x ; \sigma_{0}\right)$ is elliptic and hence

$$
\lim _{r=\infty} \inf J\left(x_{r} ; \sigma_{0}\right) \geqslant J\left(x_{0} ; \sigma_{0}\right) .
$$

Finally if $x_{r} \rightarrow x_{0}, \sigma_{r} \rightarrow \sigma_{0}, J\left(x_{r} ; \sigma_{r}\right) \rightarrow J\left(x_{0} ; \sigma_{0}\right)$, then

$$
\left|J\left(x_{r} ; \sigma_{r}\right)-J\left(x_{0} ; \sigma_{0}\right)\right| \geqslant|| J\left(x_{r} ; \sigma_{r}\right)-J\left(x_{r} ; \sigma_{0}\right)|-| J\left(x_{0} ; \sigma_{0}\right)-J\left(x_{r} ; \sigma_{0}\right)|| \text {. }
$$

As above $J\left(x_{r} ; \sigma_{r}\right) \rightarrow J\left(x_{r} ; \sigma_{0}\right)$ so that $J\left(x_{r} ; \sigma_{0}\right) \rightarrow J\left(x_{0} ; \sigma_{0}\right)$. But $J\left(x ; \sigma_{0}\right)$ is elliptic and therefore $x_{r} \Rightarrow x_{0}$. This completes the proof.

For the next theorem we will define a resolution $\{R(\lambda) \mid a \leqslant \lambda \leqslant b\}$ of a subspace $R$. That is for each $\lambda$ in $[a, b]$, let $R(\lambda)$ be a closed subspace of $R$ given by (5) such that $R(a)=0, R(b)=R, a \leqslant \lambda_{1}<\lambda_{2} \leqslant b$ implies $R\left(\lambda_{1}\right) \subset$ $R\left(\lambda_{2}\right)$. We usually require one or both of the additional hypotheses:

$$
\begin{aligned}
& R\left(\lambda_{0}\right)=\bigcap_{\lambda_{0}<\lambda \leqslant b} R(\lambda) \quad \text { whenever } a \leqslant \lambda_{0}<b ; \\
& R\left(\lambda_{0}\right)=\operatorname{cl}\left(\bigcup_{a \leqslant \lambda<\lambda_{0}} R(\lambda)\right) \quad \text { whenever } a<\lambda_{0} \leqslant b
\end{aligned}
$$

where $\operatorname{cl}(S)$ denotes the closure of the set $S$.

Theorem 9 has been given in [2, Theorem 2].

THEOREM 9. Hypothesis (19) implies (11) in the $R, \sigma$ setting. In particular (19a) implies (11a) while (19a) implies (11b). 
Thus, in particular, for the resolution spaces given by (5b) we have

Corollary 10. Hypothesis (5b) implies (11).

4. The approximation results. In this section we will show that inequality (15) can be applied in a general way to obtain an approximation focal point theory. This theory can then be applied to a multitude of approximation problems for $2 n$th order integral-differential equations of form (10).

Let $M=\Lambda \times \Sigma$ be the metric space with metric $d$ defined by $d\left(\mu_{1}, \mu_{2}\right)=$ $\left|\lambda_{2}-\lambda_{1}\right|+\rho\left(\sigma_{2}, \sigma_{1}\right)$ where $\mu_{1}=\left(\lambda_{1}, \sigma_{1}\right), \mu_{2}=\left(\lambda_{2}, \sigma_{2}\right) ;(\Sigma, \rho)$ is a metric space; and $\Lambda=[a, b]$ with the usual absolute valued metric. For each $\mu=$ $(\lambda, \sigma)$ in $M$ and $J(x ; \sigma)$ in $(18)$, define $J(x ; \mu)=J(x ; \sigma)$ on the space $C(\mu)=$ $A(\sigma) \cap B(\lambda)$. Let $s(\mu)=s(\lambda, \sigma)$ and $n(\mu)=n(\lambda, \sigma)$ denote the index (signature) and nullity of $J(x ; \mu)$ on $C(\mu)$.

In many senses Theorem 11 is the main result for applications to approximation problems. It allows us to extend (15) to more general problems. This result has been given in [3, Theorem 7].

THEOREM 11. Assume that the quadratic forms $J(x ; \sigma)$ and the spaces $A(\sigma)$ satisfy (11) and (12). For any $\mu_{0}=\left(\lambda_{0}, \sigma_{0}\right)$ in $M$ there exists $\delta>0$ such that if $\mu=(\lambda, \sigma), d\left(\mu_{0}, \mu\right)<\delta$ then

$$
s\left(\lambda_{0}, \sigma_{0}\right) \leqslant s(\lambda, \sigma) \leqslant s(\lambda, \sigma)+n(\lambda, \sigma) \leqslant s\left(\lambda_{0}, \sigma_{0}\right)+n\left(\lambda_{0}, \sigma_{0}\right) .
$$

\section{Furthermore}

(21) $n\left(\lambda_{0}, \sigma_{0}\right)=0$ implies $s(\lambda, \sigma)=s\left(\lambda_{0}, \sigma_{0}\right)$ and $n(\lambda, \sigma)=0$.

We now digress to interpret Theorem 11 for the integral-differential equations (8) or (10). For convenience we will assume $A(\mu)=B$. For each $\sigma$ in $\Sigma$ and quadratic form $J(x ; \sigma)$ given by (18) let

$$
\tau_{\beta \sigma}^{j}(t)=R_{\alpha \beta \sigma}^{i j}(t) x_{\alpha}^{i}(t)+\int_{a}^{b} K_{\alpha \beta \sigma}^{i j}(s, t) x_{\alpha}^{(i)}(s) d s,
$$

$$
v_{\beta \sigma}^{0}(t)=\int_{a}^{t} \tau_{\beta \sigma}^{0}(s) d s+c_{\beta \sigma}^{0},
$$

$$
v_{\beta \sigma}^{k}(t)=\int_{a}^{t}\left[\tau_{\beta \sigma}^{k}(s)-v_{\beta \sigma}^{k-1}\right] d s+c_{\beta \sigma}^{k}
$$

$$
\tau_{\beta \sigma}^{n}(t)=v_{\beta \sigma}^{n-1}(t)
$$

(10)o $\frac{d^{n}}{d t^{n}}\left[\tau_{\beta \sigma}^{n}(t)\right]-\frac{d^{n-1}}{d t^{n-1}}\left[\tau_{\beta \sigma}^{n-1}(t)\right]+\cdots+(-1)^{n} \tau_{\beta \sigma}^{0}(t)=0$ 
be associated with equations (6) through (10) in the obvious manner. We recall that the nullity of $Q(x)$ on $D$ is the dimension of $D_{0}$ where $D_{0}=\{x$ in $D \mid$ $Q(x, y)=0$ for all $y$ in $D\}$. Then by Theorem 1 we have

THEOREM 12. The integer $n(\lambda, \sigma)$ is the number of distinct nonzero solutions $x(t)=\left(x_{1}(t), x_{2}(t), \ldots, x_{p}(t)\right)$ to

$$
\tau_{\beta \sigma}^{n}(t)=v_{\beta \sigma}^{n-1}(t)
$$

satisfying the transversality conditions

$$
A_{\alpha \beta \sigma}^{k l} x_{\alpha}^{(k)}(a)+\mu_{\gamma \sigma} M_{\gamma \beta}-v_{\beta \sigma}^{l}(a)=0
$$

and the boundary conditions

$$
M_{\gamma \alpha}^{k} x_{\alpha}^{(k)}(a)=0, \quad x_{\alpha}^{(l)}(\lambda)=0
$$

$(\alpha, \beta=1, \ldots, p ; k, l=0, \ldots, n-1 ; \gamma=1, \ldots, m \leqslant n p)$.

We note that for $\sigma_{0}$ fixed $s\left(\lambda, \sigma_{0}\right)$ and $m\left(\lambda, \sigma_{0}\right)=s\left(\lambda, \sigma_{0}\right)+n\left(\lambda, \sigma_{0}\right)$ are nondecreasing nonnegative integer-valued functions of $\lambda$. It has been shown in [2, Theorem 6] that $s(\lambda-0, \sigma)=s(\lambda, \sigma)$ and in [2, Theorem 8] that the disjoint hypothesis of Theorem 13 implies $s(\lambda+0, \sigma)=s(\lambda, \sigma)+n(\lambda, \sigma)$. Thus $s\left(\lambda+0, \sigma_{0}\right)-s\left(\lambda-0, \sigma_{0}\right)=n\left(\lambda, \sigma_{0}\right)$. These results follow from (20). This disjoint hypothesis is usually called "normality" in problems of differential equations, calculus of variations, and control theory.

A point $\lambda$ at which $s\left(\lambda, \sigma_{0}\right)$ is discontinuous will be called a focal point of $J(x ; \sigma)$ relative to $B(\lambda)(\lambda$ in $\Lambda)$. The difference $f\left(\lambda, \sigma_{0}\right)=s\left(\lambda+0, \sigma_{0}\right)-$ $s\left(\lambda-0, \sigma_{0}\right)$ will be called the order of the focal point. A focal point will be counted the number of times equal to its order.

THEOREM 13. Assume for $\sigma_{0}$ in $\Sigma$ that $C_{0}\left(\lambda_{1}, \sigma_{0}\right) \cap C_{0}\left(\lambda_{2}, \sigma_{0}\right)=0$ when $\lambda_{1} \neq \lambda_{2}$, then $f\left(a, \sigma_{0}\right)=0, f\left(\lambda, \sigma_{0}\right)=n\left(\lambda, \sigma_{0}\right)$ on $a \leqslant \lambda \leqslant b$. Thus if $\lambda_{0}$ in $\Lambda$ the following quantities are equal:

(i) the sum $\Sigma_{a<\lambda<\lambda_{0}} n\left(\lambda, \sigma_{0}\right)$,

(ii) the signature $s\left(\lambda_{0}, \sigma_{0}\right)$ of $J(x ; \sigma)$ on $B\left(\lambda_{0}\right)$,

(iii) the sum $\Sigma s\left(\lambda_{i}+0, \sigma_{0}\right)-s\left(\lambda_{i}, \sigma_{0}\right)$ taken over all $\lambda_{i}$, such that $a \leqslant$ $\lambda_{i}<\lambda_{0}$ and $s\left(\lambda, \sigma_{0}\right)$ discontinuous at $\lambda_{i}$,

(iv) the number of focal points on $a \leqslant \lambda<\lambda_{0}$,

(v) the number of $\lambda_{i}$ and corresponding $x \neq 0$ as described in Theorem 12 with $a<\lambda_{i}<\lambda_{0}$.

For the approximation setting we can say much more. In the next two results we assume that $\sigma_{0}$ in $\Sigma$ satisfies $C_{0}\left(\lambda_{1}, \sigma_{0}\right) \cap C_{0}\left(\lambda_{2}, \sigma_{0}\right)=0$ when 
$\lambda_{1} \neq \lambda_{2}$. Since this implies that $n\left(\lambda, \sigma_{0}\right)=0$ except for a finite number of points $\lambda$ in $\Lambda$ we have

T HEOREM 14. Assume $\lambda^{\prime}$ and $\lambda^{\prime \prime}$ are not focal points of $\sigma_{0}\left(a \leqslant \lambda^{\prime}<\right.$ $\left.\lambda^{\prime \prime}<b\right)$ and $\lambda_{q}\left(\sigma_{0}\right) \leqslant \lambda_{q+1}\left(\sigma_{0}\right) \leqslant \cdots \leqslant \lambda_{q+k-1}\left(\sigma_{0}\right)$ are the $k$ focal points of $\sigma_{0}$ on $\left(\lambda^{\prime}, \lambda^{\prime \prime}\right)$. Then there exists an $\epsilon>0$ such that $\rho\left(\sigma, \sigma_{0}\right)<\epsilon$ implies $\lambda_{q}(\sigma) \leqslant \lambda_{q+1}(\sigma) \leqslant \cdots \leqslant \lambda_{q+k-1}(\sigma)$ are the $k$ focal points of $\sigma$ on $\left(\lambda^{\prime}, \lambda^{\prime \prime}\right)$.

COROllary 15. The $k$ th focal point $\lambda_{k}(\sigma)$ is a continuous function of $\sigma(k=-1,2, \ldots)$.

We now turn our attention to the case where a nonzero solution to $\tau_{\beta \alpha_{0}}^{n}(t)$ $=v_{\beta \sigma_{0}}^{n-1}(t)$ subject to (9) $\sigma$ is identically zero on a subinterval of $\Lambda$ ("abnormal" problem). In this case $f\left(\lambda, \sigma_{0}\right) \neq 0$ if and only if $\lambda$ is the right-hand endpoint of the subinterval; $s\left(b, \sigma_{0}\right)$ counts the number of such intervals. A detailed explanation of abnormal problems may be found in [2] and [3]. This phenomenon is the "focal interval" problem encountered in optimum control theory. It should be regarded as a generalized type of oscillation problem.

5. Further problems. The purpose of this section is to indicate how this theory may be applied to a variety of problems in this setting. For purposes of convenience we will designate problem areas in the following way: $(F)$ will denote the approximation theory for focal points and focal intervals discussed in $\S 4 ;(E)$ will denote the approximation theory for eigenvalues of compact operators (quadratic forms) in this setting; $(O)$, oscillation theory; $(N)$, numerical approximation. We also note that these problem areas have interesting intersections, for example: ( $F N$ ) denotes a numerical approximation theory for focal points, $(E N)$ denotes a numerical approximation theory for eigenvalues of compact operators, etc. It can be shown in many cases that our numerical theory leads to efficient numerical algorithms. In this paper we will consider additional areas $(O)$ and $(F N)$.

(O) The problem of oscillation for general Fredholm equations. While there is a great deal of mathematical literature on oscillation theory for less general problems we note that the oscillation problem as we define it is an immediate consequence of our theory on focal points. In fact we will "define" oscillation points to be the same as focal points (just preceding Theorem 13). Our definition is not the usual definition (except for normal problems with $n=1$ ) which requires a nonzero solution to vanish. In our case we will require conditions such as (9) $\sigma$ and (22) in Theorem 12. In particular we note for $2 n$th order differential equations we require not only $x(\lambda)=0$ but also $x^{(l)}(\lambda)=0$ 
$(l=1, \ldots, n-1)$. This definition is more "operable" in that it makes sense when talking about optimum problems for calculus of variations/optimum control theory, comparison theorems and Sturm separation theorems.

Thus an approximation theory for oscillations of generalized Fredholm equations is immediately obtained from Theorem 12 with the definition of oscillation in the next paragraph, the inequality results (20), and the results in Theorems 13 to 15 .

Let $\sigma$ in $\Sigma, \lambda$ in $\Lambda=[a, b]$ and assume the notation and setting of $\S 4$. For each $\sigma$ in $\Sigma$ we will say that the quadratic form $J(x ; \sigma)$ in (18), subject to the boundary conditions $M_{\gamma \alpha}^{k} x_{\alpha}^{(k)}(a)=0$, is oscillatory of degree $m$ if $s(b, \sigma)=$ $m$. Equation (8) $\sigma$ is oscillatory of degree $m$ on $a \leqslant t \leqslant b$ if there exists a nonzero solution $x(t)$ to equation (8) $\sigma$ such that the associated quadratic form $J(x ; \sigma)$, subject to the boundary conditions $M_{\gamma \alpha}^{k} x_{\alpha}^{(k)}(a)$ is oscillatory of degree $m$. In this case the vector $x(t)$ is an oscillatory solution of degree $m$ on $a \leqslant t \leqslant$ $b$. Equation (8) $\sigma$ is oscillatory if for any integer $m_{0}>0$ there exists real numbers $a<b$ such that equation (8) $\sigma$ is oscillatory of degree $m$ on $a \leqslant t \leqslant b$ and $m \geqslant m_{0}$. Finally equation (8) $\sigma$ is nonoscillatory if it is not oscillatory.

We remark that problem type $(O N)$ follows immediately from our ideas in the same way that problem type $(F N)$ follows from $(F)$ in the remainder of this paper.

$(\vec{F} N)$ The numerical focal point approximation problem. The notation will be a continuation of the notation in $\S 4$ : Let $\Psi$ denote the set of all natural numbers $\psi=1 / m(m=1,2,3, \ldots)$ and zero. The metric on $\Psi$ is the absolute value function | $\mid$. Let $Z=\Psi \times M=\Psi \times \Lambda \times \Sigma$ with metric $\theta\left(\zeta_{1}, \zeta_{2}\right)=\left|\psi_{2}-\psi_{1}\right|$ $+\rho\left(\sigma_{2}, \sigma_{1}\right)+\left|\lambda_{2}-\lambda_{1}\right|$ where $\zeta_{i}=\left(\psi_{i}, \lambda_{i}, \sigma_{i}\right)$ in $Z(i=1,2)$. We define $s(\zeta)$ $=s(\psi, \lambda, \sigma)$ to be the index (signature) of the quadratic form $J(x ; \zeta)$ on the space $C(\zeta)=B(\lambda) \cap S(\psi)$ where $S(\psi)$ and $J(x ; \zeta)$ are defined below. The nullity $n(\zeta)=n(\psi, \lambda, \sigma)$ is defined analogously in the obvious way.

For each nonzero $\psi$ in $\Psi$ the space $S(\psi)$ is the space of spline functions of degree $2 n-1$ (or order $2 n$ ) with knots at the points $\pi(\psi)=\left\{a_{k}=a+k(b-a) / m\right.$ : $k=0,1, \ldots, m ; \psi m=1\}$. The vector space $S(\psi)$ has dimension $p(m+1)$. It is the space of $\operatorname{arcs} x(t)=\left(x_{1}(t), \ldots, x_{p}(t)\right)$ where each $x_{\sigma}(t)$ is a polynomial of degree at most $2 n-1$ on each subinterval $\left(a_{k}, a_{k+1}\right)$ and such that the $(2 n-2)$ th derivative of $x_{\alpha}(t)$ is continuous on $[a, b]$.

The quadratic forms are defined from (18) in the following way. For each $\psi \neq 0$ define $A_{\alpha \beta \sigma \psi}^{k l}=A_{\alpha \beta \sigma}^{k l}, K_{\alpha \beta \sigma \psi}^{i j}(s, t)=K_{\alpha \beta \sigma}^{i j}(\bar{s}, \bar{t})$, and $R_{\alpha \beta \sigma \psi}(t)=$ $R_{\alpha \beta \sigma}(\bar{t})$ where $\bar{t}=\left(a_{k}+a_{k+1}\right) / 2$ if $a_{k} \leqslant t<a_{k+1}$ and $\bar{s}=\left(a_{k}+a_{k+1}\right) / 2$ if $a_{k} \leqslant s<a_{k+1}(k=0, \ldots, m-1)$. Finally define 


$$
\begin{aligned}
J(x ; \zeta)= & A_{\alpha \beta \sigma}^{k l} x_{\alpha}^{(k)}(a) x_{\beta}^{(l)}(a) \\
& +\int_{a}^{a} k \int_{a}^{a} k K_{\alpha \beta \sigma \psi}^{i j}(s, t) x_{\alpha}^{(j)}(s) x_{\beta}^{(j)}(t) d s d t \\
& +\int_{a}^{a} k K_{\alpha \beta \sigma \psi}^{i j}(t) x_{\alpha}^{(i)}(t) x_{\beta}^{(j)}(t) d t
\end{aligned}
$$

on the space $B(\lambda) \cap S(\psi)$ where $\lambda$ satisfies $a_{k} \leqslant \lambda<a_{k+1}, \zeta=(\psi, \lambda, \sigma)$.

For $\psi=0$ define $s(\zeta)=s(0, \lambda, \sigma)$ and $n(\zeta)=n(0, \lambda, \sigma)$ to be the index and nullity of the quadratic form $J(x ; \sigma)$ in $(18)$ restricted to $B(\lambda)$.

As above it can be shown that there exists $\delta>0$ such that $\zeta_{0}=\left(0, \lambda_{0}, \sigma_{0}\right)$ and $\zeta=(\psi, \lambda, \sigma)$ in $Z, \theta\left(\zeta, \zeta_{0}\right)<\delta$ implies

$$
\begin{aligned}
s\left(0, \lambda_{0}, \sigma_{0}\right) & \leqslant s(\psi, \lambda, \sigma) \leqslant s(\psi, \lambda, \sigma)+n(\psi, \lambda, \sigma) \\
& \leqslant s\left(0, \lambda_{0}, \sigma_{0}\right)+n\left(0, \lambda_{0}, \sigma_{0}\right) .
\end{aligned}
$$

Furthermore if the " $\sigma_{0}$ problem" is normal then $n\left(0, \lambda_{0}, \sigma_{0}\right)=0$ except on a finite set of points in $[a, b]$. This leads to the following for "normal" problems.

THEOREM 16. The kth focal point $\lambda_{k}(\sigma, \psi)$ is a continuous function of $\sigma$ and $\psi$ for $a \leqslant \lambda<b$.

It can be shown by methods similar to those in [5] that if $\psi$ is not zero and $a_{k} \leqslant \psi<a_{k+1}$ that $s(\psi, \lambda, \sigma)$ is the number of negative eigenvalues of a $(k+1) \times p$ real symmetric matrix $D(\psi, \sigma)$ which is sparse (a preponderance of zeros). For $p=1$ and $n=1$ we obtain a tridiagonal matrix. For $p=1$ we note that the elements of a basis for $S(\psi)$ have support on at most $2 n$ intervals $\left[a_{k}, a_{k+1}\right]$. Thus our matrix is in $(4 n-1)$ th "diagonal form". The number of negative eigenvalues can thus be obtained by a generalized Sturm sequence argument applied to a difference equation of the form $u_{l}=c_{1, l} u_{l-1}+c_{2, l} u_{l-2}$ $+\cdots+c_{4 n-1, l} u_{l-4 n+1}$ where $u_{l}$ is the determinant of the $l \times l$ submatrix of $D(\psi, \sigma)$ made up of the first $l$ rows and columns and $a_{l} \leqslant \psi<a_{l+1}$.

These methods are currently being applied to give an efficient, feasible algorithm when $n=1$.

6. Two specific examples. In $\$ 5$ we have indicated in a general way how our theory may be applied to a wide variety of problems. In this section we will give some specific results of current interest, namely $(O)$ the oscillation problem and $(O N)$ or $(F N)$ the numerical oscillation problem discussed in $\S 5$.

In each case we consider only second order equations but for different reasons: For oscillation the $2 n$th order case using our methods is not significantly more difficult than the second order case, if by "zero or oscillation" we mean 
the solution $x(t)$ and its $n-1$ derivatives $x^{\prime}(t), \ldots, x^{(n-1)}(t)$ are zero at a point. In our numerical example the higher order problems are numerically much more complex. However, if our example is fully understood equivalent methods should hold in the $2 n$th order case. In any case, Givens' method will transform the matrix $D(\psi, \sigma)$ associated with the $2 n$th order problem to tridiagonal form by rotation matrices and our current methods can be applied.

Once again we remind the reader that our results subsume many results and ideas for ordinary differential equations not yet obtained by more conventional methods. Furthermore, our methods provide interesting new ideas in the more "elementary" theory of linear algebra.

In our first result we will compare the oscillation properties of two differential equations:

$$
\left(r_{i}(t) x^{\prime}(t)\right)^{\prime}+p_{i}(t) x(t)=\int_{a}^{b} q_{i}(s, t) d s-\frac{d}{d t} \int_{a}^{b} l_{i}(s, t) x^{\prime}(s) d s
$$

associated with the quadratic form

$$
\begin{aligned}
J_{i}(x)= & \int_{a}^{b}\left[r_{i}(t) x^{\prime 2}(t)-p_{i}(t) x^{2}(t)\right] d t \\
& +\int_{a}^{b} \int_{a}^{b} q_{i}(s, t) x(s) x(t) d s d t \\
& +\int_{a}^{b} \int_{a}^{b} l_{i}(s, t) x^{\prime}(s) x^{\prime}(t) d s d t .
\end{aligned}
$$

In the above $i=1,2 ; r_{2}(t) \geqslant r_{1}(t)>0, p_{2}(t) \leqslant p_{1}(t) ; q_{2}(s, t)-q_{1}(s, t)=$ $u(s) u(t), l_{2}(s, t)-l_{1}(s, t)=v(s) v(t)$.

THEOREM 17. Let $a<\lambda_{1}<\lambda_{2}<\lambda_{3} \cdots<\lambda_{m_{1}} \leqslant b$ be the oscillation points associated with equation (25) and let $a<\mu_{1}<\mu_{2}<\mu_{3}<\cdots<\mu_{m_{2}}$ $\leqslant b$ be associated with equation (25) $)_{2}$. Then $m_{2} \leqslant m_{1}$ and for each $j$ such that $1 \leqslant j \leqslant m_{2}$ we have $\lambda_{j} \leqslant \mu_{j}$.

Let $x(t)$ be any vector vanishing at $a$ and $b$, then

$$
\begin{aligned}
J_{2}(x)-J_{1}(x)= & \int_{a}^{b}\left[\left(r_{2}-r_{1}\right) x^{\prime 2}(t)-\left(p_{2}-p_{1}\right) x^{2}(t)\right] d t \\
& +\left[\int_{a}^{b} u(s) x(s) d s\right]^{2}+\left[\int_{a}^{b} v(s) x^{\prime}(s) d s\right]^{2} \geqslant 0 .
\end{aligned}
$$

Thus $J_{2}(x) \geqslant J_{1}(x)$ so that a negative vector of $J_{2}(x)$ is a negative vector of $J_{1}(x)$ (i.e., $J_{2}(y)<0$ implies $\left.J_{1}(y)<0\right)$. The result now follows from Theorem 13.

The next theorem shows that the oscillation points of ordinary differential equations can be "bracketed" by oscillation points of integral differential equations and conversely. 
Corollary 18. Assume that $q_{1}(s, t)=l_{1}(s, t) \equiv 0$ in Theorem 17. Then $\lambda_{j} \leqslant \mu_{j}$ for each $j$ such that $1 \leqslant j \leqslant m_{2}$. Conversely, if $q_{1}(s, t)=l_{1}(s, t) \equiv 0$; $r_{1}(t) \geqslant r_{2}(t)>0, p_{2}(t) \geqslant p_{1}(t) ; q_{2}(s, t)=-u(s) u(t), l_{2}(s, t)=-v(s) v(t)$, then $\mu_{j} \leqslant \lambda_{j}$ for each $j$ such that $1 \leqslant j \leqslant m_{1}$.

The above inequalities are "sharp." For example, assume the conditions of Theorem 17, then from an argument similar to Theorem 21 below we have

CoRollary 19. Let $j$ be a nonnegative integer such that $r_{2}(t)-r_{1}(t)>$ 0 on $\left[a, \mu_{j}\right] ; p_{1}(t)-p_{2}(t)>0$ on $\left[a, \mu_{j}\right] ;$ or $\int_{a}^{\mu_{j}} u(s) x(s) d s \neq 0$ or $\int_{a}^{\mu_{j}} v(s) x(s) d x$ $\neq 0$ where $x(s)$ is a solution to $(25)_{2}$ on $\left[a, \mu_{j}\right]$ vanishing on $\left[\mu_{j}, b\right]$. Then $\lambda_{j}$ $<\mu_{j}$.

Theorem 20 (Sturm Separation Theorem). If $x_{1}(t)$ and $x_{2}(t)$ are two linearly independent solutions of (25), then between any two consecutive zeros of $x_{1}(t)$ there is a zero of $x_{2}(t)$.

The negation implies there exist points $a<a_{1}<a_{2}<a_{3}$ such that if $s(t)$ is the signature of the appropriate quadratic form we have $s\left(a_{2}+\right)-s\left(a_{1}-\right)>0$ and $s\left(a_{3}-\right)-s(a)=0$ which is clearly impossible.

A final result is on disconjugacy type theorems, thus

ThEOREM 21. Assume the hypothesis of Theorem 17 except that $q_{2}(s, t)$ $\geqslant q_{1}(s, t)$. Then $\lambda_{1} \leqslant \mu_{1}$ if $\mu_{1}$ exists. Furthermore, $\lambda_{1}<\mu_{1}$ unless $r_{1}(t) \equiv$ $r_{2}(t), p_{1}(t) \equiv p_{2}(t)$ and $q_{1}(s, t) \equiv q_{1}(s, t)$ on $\left[a, \mu_{1}\right]$ and $\int_{a}^{\mu_{1}} v(s) x(s) d s=0$ for any solution to $(25)_{2}$ on $\left[a, \mu_{1}\right]$.

By hypothesis if $x(t)$ is any vector vanishing at $t=a$, and in the interval $\left[\mu_{1}, b\right]$, and $x\left(t_{1}\right) x\left(t_{2}\right) \geqslant 0$ en $\left[a, \mu_{1}\right]$, then

$$
\begin{aligned}
J_{2}(x)-J_{1}(x)= & \int_{a}^{\mu_{1}}\left[\left(r_{2}-r_{1}\right) x^{\prime 2}-\left(p_{2}-p_{1}\right) x^{2}(t)\right] d t \\
& +\int_{a}^{\mu_{1}} \int_{a}^{\mu_{1}}\left[q_{2}(s, t)-q_{1}(s, t)\right] x(s) x(t) d s d t \\
& +\left[\int_{a}^{\mu_{1}} v(s) x^{\prime}(s) d s\right]^{2} \geqslant 0 .
\end{aligned}
$$

The result again follows by Theorem 13 .

Conversely, if $x_{0}(t)$ is any solution to $(25)_{2}$ with $x_{0}(a)=0$ then $J_{2}(x)$ "restricted" to $\left[a, \mu_{1}\right]$ has value zero. If $J_{2}\left(x_{0}\right)-J_{1}\left(x_{0}\right)>0$ restricted to $\left[a, \mu_{1}\right]$ then by Theorem 13 we have $\lambda_{1}<\mu_{1}$.

We now turn our attention to the numerical oscillation problem which is a continuation of the ideas in ( $F N)$ of the previous section. Full details including proofs and sample runs will appear elsewhere. We remark that sample runs to 
numerically solve second-order differential equations indicate that our methods are faster and more accurate than "in-house" numerical subroutines.

For convenience we will restate our problem: Thus let

$$
\left(r(t) x^{\prime}(t)\right)^{\prime}+p(t) x(t)=\int_{a}^{b} q(s, t) d s-\frac{d}{d t} \int_{a}^{b} l(s, t) x^{\prime}(s) d s
$$

be the Euler-Lagrange equation for the quadratic form

$$
\begin{aligned}
J(x) & =\int_{a}^{b}\left[r(t) x^{\prime 2}(t)-p(t) x^{2}(t)\right] d t \\
& +\int_{a}^{b} \int_{a}^{b} q(s, t) x(s) x(t) d s d t+\int_{a}^{b} \int_{a}^{b} l(s, t) x^{\prime}(s) x^{\prime}(t) d s d t .
\end{aligned}
$$

Following the ideas and methods in (FN) we set $n=1$ and for fixed $m$ (a "large" positive integer) we set $\psi=1 / m$ and $\pi(\psi)=\left\{a_{0}=a<a_{1}<\cdots\right\}$. Let $y_{k}(t)$ be the spline hat function of degree 1 on $\left[a_{k-1}, a_{k+1}\right]$; that is $y_{k}(t)$ $=1-m\left|a_{k}-t\right|$ if $a_{k-1} \leqslant t \leqslant a_{k+1}$ and $y_{k}(t)^{\circ} \equiv 0$ otherwise. Following these ideas if $x(t)=A_{k} y_{k}(t)$ where repeated indices are summed, then our approximation for $J(x)$ in (27) is given by (23) which we write as the quadratic form

$$
J(x ; \zeta)=A_{k} J\left(y_{k}, y_{l}\right) A_{l}
$$

The matrix $J\left(y_{k}, y_{l}\right)$ "is" the tridiagonal matrix $D(\psi, \sigma)$, and $s(\psi, \lambda, \sigma)$ in (24) represents the number of negative vectors of $J(x ; \zeta)$ restricted to the piecewise linear functions $x(t)=A_{k} y_{k}(t)$ defined on $\left[a, a_{k}\right]$ where $\lambda$ satisfies $a_{k} \leqslant \lambda$ $<a_{k+1}$ and $D(\psi, \sigma)$ has been tridiagonalized by Givens' method.

We now give a simple but elegant algorithm to find the "Euler-Lagrange equation" of the matrix $D=\left(d_{i j}\right)=D(\psi, \sigma)$. This result is actually motivated by our concept of negative vector. That is, intuitively, given $D$ we will construct a vector $c=\left(c_{1}, c_{2}, c_{3}, \ldots\right)^{T}$ so that (i) if $C=c_{k} y_{k}$ then $C D C^{T}$ is as "negative as possible"; (ii) if the numbers $c_{k}$ "change sign" at $k=m_{1}, m_{2}, \ldots$, then the vectors $C_{1}(t)=c_{1} y_{1}+\cdots+c_{m_{1}} y_{m_{1}}, C_{2}(t)=c_{m_{1}+1} y_{m_{1}+1}+\cdots+$ $c_{m_{2}} y_{m_{2}}$, etc., are linearly independent negative vectors, i.e., $C_{1} D C_{1}^{T}<0$, etc.; (iii) the vectors $C_{1}, C_{2}, C_{3}, \ldots$ form a basis for the negative space of $D$; (iv) if $z(t)$ is a solution to (26), $c_{1}=z\left(a_{1}\right)$ and $m$ is large then $z(t)$ is approximated by $C(t)$ in the mean squared norm, i.e.,

$$
\int_{a}^{b}\left(C^{\prime}(t)-z^{\prime}(t)\right)^{2} d t \rightarrow 0 \quad \text { as } m \rightarrow \infty .
$$

To define the numbers $c_{1}, c_{2}, c_{3}, \ldots$ we proceed as follows: let $d_{i j}$ be the $(i-j)$ th element of the tridiagonal matrix $D$ given above, let $c_{1}=1$ and define recursively $c_{2}, c_{3}, c_{4}, \ldots, c_{k+1}$, by

$$
d_{11} c_{1}+d_{12} c_{2}=0
$$

$$
d_{k, k-1} c_{k-1}+d_{k, k} c_{k}+d_{k, k+1} c_{k+1}=0 \text { for } k=2,3,4, \ldots
$$


Note that this procedure forces the first $k$ components of the product $D C^{T}$ to be zero and thus $C D C^{T}$ to be "as negative as possible."

Since $r(t)>0$ in (27) we may choose $m$ large enough so that $d_{i i}>0$, $d_{i i+1}<0$. We ask the interested reader to verify the following.

THEOREM 22. Let $C_{1}=\left(c_{1}, c_{2}, \ldots, c_{m_{1}}, 0,0,0, \ldots\right)$ be given with $c_{1}, c_{2}, c_{3}, \ldots$ defined by (28) and $m_{1}$ first integer such that $c_{i} c_{i+1}>0$ is not true. Then $C_{1} D C_{1}^{T}<0$. Conversely, if $k<m_{1}$ and $E=\left(c_{1}, c_{2}, \ldots, c_{k}, 0\right.$, $0,0, \ldots)$ then $E D E^{T}>0$.

The obvious analogous statements for the vectors $C_{2}, C_{3}, \ldots$ defined above can be demonstrated. More difficult is to show that this procedure counts the number of negative eigenvalues in $D$. This has been done by the author by showing that the sequence $\left\{c_{k}\right\}$ changes sign if and only if the appropriate Sturm sequence for the number of negative eigenvalues of $D$ change sign. Finally, the most difficult result, that is (iv) above

$$
\int_{a}^{b}\left(C^{\prime}(t)-z^{\prime}(t)\right)^{2} d t \rightarrow 0 \quad \text { as } m \rightarrow \infty,
$$

is established by use of our fundamental hypotheses (11) and (12).

\section{REFERENCES}

1. J. Gregory, An approximation theory for elliptic quadratic forms on Hilbert spaces: Application to the eigenvalue problem for compact quadratic forms, Pacific J. Math. 37 (1970), 383-395. MR 46 \# 2449.

2. - A theory of focal points and focal intervals for an elliptic quadratic form on a Hilbert space, Trans. Amer. Math. Soc. 157 (1971), 119-128. MR 43 \# 3878.

3. - An approximation theory for focal points and focal intervals, Proc. Amer. Math. Soc. 32 (1972), 477-483. MR 45 \# 5847.

4. - A theory of numerical approximation for elliptic forms associated with second order differential systems. Application to eigenvalue problems, J. Math. Anal. Appl. 38 (1972), 416-426. MR 48 \# 1014.

5. J. Gregory and F. Richards, Numerical approximation for 2 mth order differential systems via splines, Rocky Mountain J. Math. 5 (1975).

6. M. R. Hestenes, Applications of the theory of quadratic forms in Hilbert space to the calculus of variations, Pacific J. Math. 1 (1951), 525-581. MR 13, 759.

1966. MR 34 \# 3390.

7. Calculus of variations and optimal control theory, Wiley, New York,

8. G. C. Lopez, Quadratic variational problems involving higher order ordinary derivates, Dissertation, University of California, Los Angeles, 1961.

9. E. Y. Mikami, Focal points in a control problem, Pacific J. Math. 35 (1970), 473-485. MR 43 \# 6800 .

10. M. Morse, The calculus of variations in the large, Amer. Math. Soc. Colloq. Publ., vol. 18, Amer. Math. Soc., Providence, R. I., 1934.

DEPARTMENT OF MATHEMATICS, SOUTHERN ILLINOIS UNIVERSITY AT CARBONDALE, CARBONDALE, ILLINOIS 62901

DEPARTMENT OF MATHEMATICS, SAN DIEGO STATE UNIVERSITY, SAN DIEGO, CALIFORNIA 92115 